\section{Preliminary investigation on multiple-locus variable number tandem repeat analysis profiles of Listeria monocytogenes isolates from pork meat tested from packaging to fork}

\author{
Alessandra De Cesare, ${ }^{1}$ Antonio Parisi, 2 \\ Marta Caruso, ${ }^{2}$ Frédérique Pasquali, \\ Gerardo Manfreda'
}

'Dipartimento di Scienze e Tecnologie Agro-Alimentari, Alma Mater Studiorum

- Universita di Bologna; 'Istituto

Zooprofilattico Sperimentale della Puglia e della Basilicata, Foggia, Italy

\section{Abstract}

Listeria monocytogenes is recognised as a public health issue and a serious challenge for the food industry. L. monocytogenes strain characterisation on the basis of serotyping and molecular typing methods is used for surveillance, epidemiological tracking and outbreak investigation purposes. Genetic variants of $L$. monocytogenes have diversified into four major phylogenetic lineages, with lineages 1 and 2 each containing multiple clonal groups of public health importance. Standardised tools for easy identification of clonal groups are needed to trace such groups and determine their presence in a large variety of sources. Given the current limitations of available methods for L. monocytogenes strain typing, a potentially useful approach is multiple locus variable number of tandem repeats (VNTR) analysis (MLVA). In this study, MLVA has been applied to a random group of $82 \mathrm{~L}$. monocytogenes strains isolated from 8 different batches of loin chops obtained from the same facility and tested between packaging and consumption time. The strains typed were classified into 10 MLVA profiles containing a number of isolates ranging between 1 to 20 . According to the identified MLVA profiles, $75.6 \%$ of the pork isolates belonged to the phylogenetic lineage 2 and serotype $1 / 2 c$, frequently associated to food isolates. However, 3 pork strains belonged to the phylogenetic lineage 1 and serotype $4 \mathrm{~b}$. Moreover, 17 isolates were classified in the phylogenetic lineages 2 and serotype 1/2a. Both serotypes $4 \mathrm{~b}$ and $1 / 2 \mathrm{a}$ are frequently associated to human isolates of $L$. monocytogenes. These preliminary results show how the MLVA profiles can support the assessment of the risk profile of food products based on the contaminating $L$. monocytogenes strain types.

\section{Introduzione}

La listeriosi è una tossinfezione alimentare causata da Listeria monocytogenes. Le forme invasive di listeriosi umana includono setticemia, meningiti e infezioni materno-fetali (Lorber, 1997). Molti paesi hanno organizzato un sistema di sorveglianza per L. monocytogenes a causa della sua importanza per la salute umana ma anche perché il suo controllo è una grossa sfida per le aziende alimentari. La tipizzazione di L. monocytogenes a livello di ceppo viene utilizzata nelle attività di sorveglianza, negli studi epidemiologici e durante gli episodi tossinfettivi (Kathariou, 2002; Swaminathan e Gerner-Smidt, 2007). Studi filogenetici hanno identificato nella popolazione di $L$. monocytogenes 4 distinte linee filogenetiche delle quali le linee 1 e 2 contengono genotipi più importanti per la loro patogenicità per l'uomo (Kathariou, 2003; Nightingale et al., 2005; Orsi et al., 2011; Piffaretti et al., 2008; Ward et al., 2010). Per identificare tali genotipi e le loro fonti di contaminazione sono necessarie metodiche di tipizzazione standardizzate; tra tali metodiche, la sierotipizzazione (Seeliger e Höhne, 1979) e la polymerase chain reaction (PCR) sierotipizzazione (Doumith et al., 2004), sebbene largamente utilizzate per la caratterizzazione di $L$. monocytogenes non sono abbastanza discriminanti da poter essere impiegate nel contesto di un'analisi epidemiologica durante un focolaio di tossinfezione alimentare. L'elettroforesi in campo elettrico pulsato (PFGE) ha rappresentato per anni la metodica di riferimento per la tipizzazione di L. monocytogenes ed è ancora ampiamente utilizzata per le attività di sorveglianza e le analisi epidemiologiche (Graves e Swaminathan, 2001). Tuttavia, la sua esecuzione è molto laboriosa e richiede una notevole standardizzazione. Inoltre, è scarsamente riproducibile tra laboratori diversi. La multi-locus sequence typing (MLST) è la tecnica attualmente più utilizzata per le analisi epidemiologiche e lo studio delle popolazioni. Infatti, il profilo MLST può essere facilmente confrontato tra laboratori diversi e il dato fornito, basato sul sequenziamento, è correlabile al livello di diversità genetica ed alla frequenza di ricombinazione tra ceppi (Feil, 2004; Maiden, 2006). Tuttavia, la MLST è una metodica lunga, costosa e per $L$. monocytogenes ha un potere discriminatorio limitato (Ragon et al., 2008; den Bakker et al., 2010).

Per superare le limitazioni associate alle tecniche descritte un approccio alternativo è rappresentato dall'analisi multilocus del numero variabile di sequenze tandem (MLVA) già utilizzata per le analisi epidemiologiche di altri batteri patogeni (Lindstedt, 2005) perchè è relativamente semplice, economica e dotata di un buon potere discriminatorio. La metodi-
Correspondence: Alessandra De Cesare, Dipartimento di Scienze e Tecnologie AgroAlimentari, Alma Mater Studiorum - Università di Bologna, viale G. Fanin 50, 40127 Bologna, Italy. Tel. +39.051.2097853 - Fax: +39.051.2097852. E-mail: alessandra.decesare@unibo.it

Key words: Listeria monocytogenes, Pork cuts, MLVA, Genotyping.

Conflict of interests: the authors declare no potential conflict of interests.

Received for publication: 20 May 2013.

Revision received: 26 August 2013.

Accepted for publication: 29 August 2013.

This work is licensed under a Creative Commons Attribution 3.0 License (by-nc 3.0).

(C) Copyright A. De Cesare et al., 2014

Licensee PAGEPress, Italy

Italian Journal of Food Safety 2014; 3:1660

doi:10.4081/ijfs.2014.1660

ca MLVA si basa sullo studio della variabilità del numero di sequenze tandem in specifici loci del genoma batterico. Cinque schemi MLVA sono stati simultaneamente sviluppati per la tipizzazione di $L$. monocytogenes ed il loro impiego è già stato dimostrato in vari studi epidemiologioci (Balandyte et al., 2011; Chen et al., 2011; Houhoula et al., 2012; Li et al., 2013; Lunestad et al., 2012). I cinque schemi si differenziano per il numero di multiple locus variable number of tandem repeats (VNTR) considerato, variabile da 3 a 10.

La carne di maiale è la tipologia di carne fresca più consumata in Europa (Devine, 2003) ed il suo controllo in termini di contaminazione con microrganismi patogeni è fondamentale sia per la salute pubblica sia per le aziende alimentari. In questo studio una metodica MLVA basata sull'analisi di 6 loci VNTR è stata utilizzata per tipizzare un gruppo di 82 ceppi di L. monocytogenes, isolati da 8 lotti di carne di maiale testati dal momento del confezionamento al consumo, per valutarne la diversità $\mathrm{e}$ potenziale patogenicità per l'uomo in funzione della appartenenza alle linee filogenetiche note per $L$. monocytogenes.

\section{Materiali e Metodi}

Otto lotti di lonza di maiale sono stati campionati da Dicembre 2010 a Ottobre 2011 nello stesso laboratorio di sezionamento. In ogni lotto, la presenza/assenza di $L$. monocytogenes è stata valutata in 20 unità campionarie utilizzando la metodica IS011290-1:1996/Amd1: 2004 (ISO, 2004). Le 20 unità campionarie sono state suddivise come segue: 5 sono state 
analizzate dopo il confezionamento sotto vuoto; 5 dopo trasporto in automezzo refrigerato aziendale e stoccaggio nel banco frigo presente nel punto vendita fino al quarto giorno post confezionamento; 5 dopo trasporto e stoccaggio al punto vendita, trasporto per $45 \mathrm{~min}$ in auto senza refrigerazione e stoccaggio a $6^{\circ} \mathrm{C}$ fino al termine della shelf life del prodotto al giorno sette post-confezionamento; 5 dopo trasporto e stoccaggio al punto vendita, trasporto per $45 \mathrm{~min}$ in auto senza refrigerazione e stoccaggio a $14^{\circ} \mathrm{C}$ fino al giorno sette postconfezionamento.

Da 3 a 15 isolati sono stati selezionati a caso dai campioni positivi appartenenti ad uno stesso lotto, purificati mediante tre passaggi seriali in piastre di Brain Heart Infusion Agar (Oxoid, Milano, Italy) incubate a $37^{\circ} \mathrm{C}$ per 24 ore, identificati come $L$. monocytogenes 0 Listeria spp. con PCR mediante il protocollo di Wesley et al. (2001). Gli isolati identificati come $L$. monocytogenes sono stati genotipizzati mediante MLVA. Un totale di 6 VNTR sono stati ottimizzati per l'amplificazione in due distinte multiplex-PCR. I prodotti di reazione sono stati diluiti e miscelati in una mix contenente formammide e Genescan $600 \mathrm{Liz}$ come marcatore di peso molecolare. Le corse elettroforetiche sono state eseguite mediante 3130 Genetic Analyzer (Applied Biosystems, Carlsbad, CA, USA). I dati sono stati analizzati mediante GeneMapper $4.0 \quad$ (Applied Biosystems), i singoli alleli normalizzati, importati in Bionumerics 6.5 (Applied Maths, Sint-Martens-Latem, Belgium) ed utilizzati per l'analisi filogenetica.

\section{Risultati}

Tutte le tipologie di campioni analizzate sono risultate positive per $L$. monocytogenes. Tuttavia, i dati di prevalenza e concentrazione del patogeno nei campioni testati sono fuori dallo scopo di questo lavoro.

Complessivamente sono stati tipizzati mediante MLVA 82 ceppi isolati da prodotti al momento del confezionamento $(\mathrm{N}=16)$, dopo trasporto e stoccaggio al punto vendita $(\mathrm{N}=21)$ e dopo stoccaggio fino al giorno sette post-confezionamento a $6 \quad(\mathrm{~N}=22) \quad$ o $14^{\circ} \mathrm{C} \quad(\mathrm{N}=23)$ (Tabella 1). Nel lotto 1, gli isolati analizzati con MLVA sono stati raccolti fino al giorno 4 post confezionamento mentre nei lotti 7 ed 8 da quel momento in poi. Al contrario, negli altri cinque lotti i ceppi da tipizzare sono stati raccolti dal confezionamento al consumo. I ceppi analizzati erano classificati in 10 profili MLVA siglati da I a X, contenenti ognuno un numero di isolati variabile da 1 a 20 (Tabella 1). I lotti 1 e 3 sono stati caratterizzati da isolati con il medesimo profilo MLVA, mentre i restanti 6 lotti hanno mostrato profili MLVA diversi

Tabella 1. Profili dell'analisi multilocus del numero variabile di sequenze tandem associati agli isolati testati.

\begin{tabular}{|c|c|c|c|c|c|}
\hline \multirow[b]{2}{*}{ Lotto } & \multicolumn{5}{|c|}{ Profilo MLVA (n. isolati) } \\
\hline & Data & $t=0$ giorni & $\mathrm{t}=4$ giorni & $\mathrm{t}=7$ giomi $\left(6^{\circ} \mathrm{C}\right)$ & $\mathrm{t}=7$ giomi $\left(14^{\circ} \mathrm{C}\right)$ \\
\hline 1 & Dicembre 2010 & VII (2) & VII (1) & - & - \\
\hline 2 & Gennaio 2011 & $\begin{array}{c}\text { III (1) } \\
\text { VIII (1) }\end{array}$ & $\begin{array}{c}\text { III (1) } \\
\text { VIII (2) }\end{array}$ & $\begin{array}{l}\text { III (1) } \\
\text { IV (2) }\end{array}$ & $\begin{array}{l}\text { III (2) } \\
\text { VII (2) }\end{array}$ \\
\hline 3 & Febbraio 2011 & VII (3) & VII (4) & VII (4) & VII (4) \\
\hline 4 & Aprile 2011 & VII (4) & $\begin{array}{l}\text { VII (3) } \\
\text { VI (1) }\end{array}$ & $\begin{array}{c}\text { VII (3) } \\
\text { X (1) }\end{array}$ & VII (4) \\
\hline 5 & Maggio 2011 & VIII (2) & VIII (2) & $\begin{array}{l}\text { VIII (4) } \\
\text { VII (1) }\end{array}$ & VIII (3) \\
\hline 6 & Agosto 2011 & $\begin{array}{c}\text { VIII (1) } \\
\text { V (1) IX (1) }\end{array}$ & VIII (1)V (3) & $\begin{array}{l}\text { VIII (1) } \\
\text { V (2) }\end{array}$ & $\begin{array}{c}\text { VIII (1) } \\
\text { V (2) } \\
\text { I (1) }\end{array}$ \\
\hline 7 & Settembre 2011 & - & VII (1) & VII (2) & $\begin{array}{l}\text { VII (1) } \\
\text { VIII (1) }\end{array}$ \\
\hline 8 & Ottobre 2011 & - & $\begin{array}{c}\text { II (1) } \\
\text { VIII (1) }\end{array}$ & $\begin{array}{c}\text { II (1) } \\
\text { III (1) }\end{array}$ & $\mathrm{V}(1)$ \\
\hline Totale & & 16 & 21 & 22 & 23 \\
\hline
\end{tabular}

MLVA, analisi multilocus del numero variabile di sequenze tandem.

durante la shelf-life. In particolare, in tutti i lotti è stato osservato un profilo MLVA persistente (es. profilo III nel lotto 2, VII nel lotto 4, VIII nei lotti 5 e 6 , VII nel lotto 7 e parzialmente II nel lotto 8) e profili MLVA associati a fasi specifiche.

Come osservato in studi precedenti, i profili MLVA consentono, con una notevole affidabilità, di prevedere la linea filogenetica, il sierotipo ed il sequence type (ST) degli isolati analizzati. In questo studio, i profili MLVA dal VI al X identificati nel 75.6\% degli isolati testati indicano l'appartenenza di tali ceppi alla linea filogenetica 2 ed al sierotipo 1/2c, spesso associato a ceppi isolati da alimenti ma non dall'uomo. Al contrario, 3 isolati con profili MLVA I e IV risultano appartenere alla linea filogenetica 1 e sierotipo 4b, spesso identificato in ceppi responsabili di episodi di infezione umana. Infine, 17 isolati con profili MLVA II, III e V risultano associabili linea filogenetica 2 ed al sierotipo 1/2a, anch'esso tipico di ceppi responsabili di infezione umana.

\section{Discussione}

L. monocytogenes è uno dei patogeni frequentemente associati al consumo di carne di maiale come dimostrato da Fosse e collaboratori (2008); inoltre, Matargas e collaboratori (2008) hanno confermato la relazione tra il rischio di contrarre listeriosi ed il consumo di carne di maiale poco cotta 0 processata e da riscaldare prima del consumo. Sulla base di queste considerazioni la carne di maiale cruda, come quella analizzata in questo studio, può essere fonte di contaminazioni crociate $\mathrm{e}$ pertanto il suo controllo diventa rilevante in termini epidemiologici. Per capire il livello di rischio associato ad un prodotto alimentare contaminato con $L$. monocytogenes è importante capire come cambia la popolazione del patogeno durante la vita commerciale del prodotto. Alcune delle fasi che impattano non solo sulla sopravvivenza e moltiplicazione di L. monocytogenes nella carne fresca ma anche sulla composizione della popolazione contaminante sono la fase di manipolazione nel laboratorio di sezionamento; il trasporto e lo stoccaggio del prodotto confezionato nel punto vendita; il trasporto da parte del consumatore dal punto vendita a casa e lo stoccaggio in frigorifero prima del consumo. In questa ricerca l'impatto della fase di stoccaggio in frigorifero da parte del consumatore è stato valutato considerando come temperatura media dei frigoriferi di casa $6^{\circ} \mathrm{C}$ e come temperatura media di abuso $14^{\circ} \mathrm{C}$.

La tecnica MLVA si distingue da altre metodiche di tipizzazione utilizzate per L. monocytogenes, come la sierotipizzazione, la PFGE e la MLST, per la notevole processività e per i costi contenuti di esecuzione. Inoltre, è facilmente standardizzabile e riproducibile tra laboratori, garantendo la possibilità di confrontare gli isolati con un sistema univoco di interpretazione. I risultati ottenuti in questo studio preliminare hanno dimostrato che in funzione del profilo MLVA la maggior parte degli isolati testati ricadeva in un genotipo raramente coinvolto in episodi clinici. Tuttavia, i tre ceppi classificati nei profili MLVA I e IV potrebbero essere altamente patogeni perché associati alla linea filogenetica 1 e sierotipo 4b, ed anche i profili MLVA II, III e V del sierotipo 1/2a andrebbero ulteriormente caratterizzati a causa dell'incremento atteso delle segnalazioni relative al coinvolgimento di tali isolati in focolai di listeriosi anche nel nostro paese (Mammina et al., 
2013). Anche se la loro presenza nel prodotto post confezionamento non può essere esclusa per il basso numero di isolati tipizzati, è interessante notare come queste tipologie di ceppi compaiano alla fine della shelf life del prodotto. La maggior parte dei profili MLVA persistenti nei lotti analizzati non sembrano associabili a sierotipi altamente patogeni per l'uomo ma la loro coesistenza con ceppi potenzialmente patogeni è stata osservata nei lotti 2,6 e 8 . Questo risultato dimostra che la corretta valutazione del numero di ceppi da sottoporre a tipizzazione è fondamentale per consentire una corretta mappatura della popolazione del patogeno.

\section{Conclusioni}

La tecnica di MLVA applicata in questo studio riteniamo possa rappresentare un valido strumento diagnostico specialmente nello studio di situazioni epidemiologiche complesse, come possono essere gli stabilimenti di produzione, in cui spesso diverse popolazioni della stessa specie possono convivere e contaminare gli alimenti, ma soprattutto quando vi sia la necessità di collegare tra loro diversi episodi clinici o questi alle rispettive fonti di contaminazione. In questo studio l'applicazione dell'MLVA ha permesso di identificare nel prodotto al momento del consumo tre ceppi potenzialmente patogeni per l'uomo con sierotipo 4b in due dei lotti analizzati, confermando la necessità di caratterizzare geneticamente i ceppi di $L$. monocytogenes al fine di una efficace valutazione del rischio.

\section{Bibliografia}

Balandyte L, Brodard I, Frey J, Oevermann A, Abril C, 2011. Ruminant rhombencephalitis-associated Listeria monocytogenes alleles linked to a multilocus variablenumber tandem-repeat analysis complex. Appl Environ Microb 77:8325-35.

Chen S, Li J, Saleh-Lakha S, Allen V, Odumeru J, 2011. Multiple-locus variable number of tandem repeat analysis (MLVA) of Listeria monocytogenes directly in food samples. Int J Food Microbiol 148:8-14.

den Bakker HC, Fortes ED, Wiedmann M, 2010. Multilocus sequence typing of outbreakassociated Listeria monocytogenes isolates to identify epidemic clones.
Foodborne Pathog Dis 7:257-65.

Devine R, 2003. Meat consumption trends in the world and the European Union. Prod Anim 16:325-7.

Doumith M, Buchrieser C, Glaser P, Jacquet C, Martin P, 2004. Differentiation of the major Listeria monocytogenes serovars by multiplex PCR. J Clin Microbiol 42:381922.

Feil EJ, 2004. Small change: keeping pace with microevolution. Nat Rev Microbiol 2:48395.

Fosse J, Seegers H, Magras C, 2008. Foodborne zoonoses due to meat: a quantitative approach for a comparative risk assessment applied to pig slaughtering in Europe. Vet Res 39:1.

Graves LM, Swaminathan B, 2001. PulseNet standardized protocol for subtyping Listeria monocytogenes by macrorestriction and pulsed-field gel electrophoresis. Int J Food Microbiol 65:55-62.

Houhoula DP, Peirasmaki D, Konteles SJ, Kizis D, Koussissis S, Bratacos M, Poggas N, Charvalos E, Tsakris A, Papaparaskevas J, 2012. High level of heterogeneity among Listeria monocytogenes isolates from clinical and food origin specimens in Greece. Foodborne Pathog Dis 9:848-52.

ISO, 2004. Modification of the isolation media and the haemolysis test, and inclusion of precision data. Norma ISO 112901:1996/Amd 1:2004. International Standardization Organization ed., Ginevra, Svizzera.

Kathariou S, 2002. Listeria monocytogenes virulence and pathogenicity, a food safety perspective. J Food Protect 65:1811-29.

Kathariou S, 2003. Foodborne outbreaks of listeriosis and epidemic associated lineages of Listeria monocytogenes. In: Torrence ME and Isaacson RE, eds. Microbial food safety in animal agriculture. Iowa State University Press, Ames, IA, pp 243-256.

Li X, Huang B, Eglezos S, Graham T, Blair B, Bates J, 2013. Identification of an optimized panel of variable number tandemrepeat (VNTR) loci for Listeria monocytogenes typing. Diagn Micr Infec Dis 75:2036.

Lindstedt BA, 2005. Multiple-locus variable number tandem repeats analysis for genetic fingerprinting of pathogenic bacteria. Electrophoresis 26:2567-82.

Lorber B, 1997. Listeriosis. Clin Infect Dis 24:19.

Lunestad BT, Truong TT, Lindstedt BA, 2012. A multiple-locus variable number tandem repeat analysis (MLVA) of Listeria monocytogenes isolated from Norwegian salmon-processing factories and from listeriosis patients. Epidemiol Infect 141:2101-10.

Maiden MC, 2006. Multilocus sequence typing of bacteria. Annu Rev Microbiol 60:561-88.

Mammina C, Parisi A, Guaita A, Aleo A, Bonura C, Nastasi A, Pontello M, 2013. Enhanced surveillance of invasive listeriosis in the Lombardy region, Italy, in the years 20062010 reveals major clones and an increase in serotype 1/2a. BMC Infect Dis 13:152.

Mataragas M, Skandamis PN, Drosinos EH, 2008. Risk profiles of pork and poultry meat and risk ratings of various pathogen/product combinations. Int J Food Microbiol 126:1-12.

Nightingale KK, Windham K, Wiedmann M, 2005. Evolution and molecular phylogeny of Listeria monocytogenes isolated from human and animal listeriosis cases and foods. J Bacteriol 187:5537-51.

Orsi RH, den Bakker HC, Wiedmann M, 2011. Listeria monocytogenes lineages: Genomics, evolution, ecology, and phenotypic characteristics. Int J Med Microbiol 301:79-96.

Piffaretti JC, Kressebuch H, Aeschbacher M, Bille J, Bannerman E, Ragon M, Wirth T, Hollandt F, Lavenir R, Lecuit M, Le Monnier A, Brisse S, 2008. A new perspective on Listeria monocytogenes evolution. PLoS Pathog 4:e1000146.

Ragon M, Wirth T, Hollandt F, Lavenir R, Lecuit M, Le Monnier A, Brisse S, 2008. A new perspective on Listeria monocytogenes evolution. PLoS Pathog 4:e1000146.

Seeliger HPR, Höhne K, 1979. Serotyping of Listeria monocytogenes and related species. Method Microbiol 13:31-49.

Swaminathan B, Gerner-Smidt P, 2007. The epidemiology of human listeriosis. Microbes Infect 9:1236-43.

Ward TJ, Usgaard T, Evans P, 2010. A targeted multilocus genotyping assay for lineage, serogroup, and epidemic clone typing of Listeria monocytogenes. Appl Environ Microb 76:6680-4.

Wesley IV, Harmon KM, Dickson JS, Schwartz AR, 2001. Application of a multiplex polymerase chain reaction assay for the simultaneous confirmation of Listeria monocytogenes and other Listeria species in turkey sample surveillance. J Food Protect 65:780-5. 